\title{
Développement de la technologie des fonds de sauces en utilisant les coproduits issus de la production du Kilishi
}

\author{
Stéphanie C. W. TIENDREBEOGO ${ }^{1,2}$, Donatien KABORE ${ }^{2 *}$, Abel TANKOANO ${ }^{2}$, \\ Adama PARE ${ }^{2}$, Fatoumata HAMA-BA ${ }^{2}$, Amanda T.J.A. DOUAMBA ${ }^{2,3}$, \\ Mamoudou H. DICKO ${ }^{1}$ et Hagrétou SAWADOGO-LINGANI ${ }^{2}$
}

\footnotetext{
${ }^{1}$ Université Joseph Ki-Zerbo, Unité de Formation et de Recherche en Sciences de la Vie et de la Terre, Laboratoire de Biochimie Biotechnologie Technologie Alimentaire et Nutrition (LABIOTAN) 09 BP 848 Ouagadougou 09, Burkina Faso 09.

${ }^{2}$ Centre National de la Recherche Scientifique et Technologique, Institut de Recherche en Sciences Appliquées et Technologies, Département Technologie Alimentaire, 03 BP 7047, Ouagadougou 03, Burkina Faso.

${ }_{3}$ Université Saint Thomas d'Aquin, Faculté des Sciences et Technologie, Agrosylvopastorale option Productions Animales, 06 BP 10212 Ouagadougou 06, Burkina Faso.

*Auteur correspondant ; E-mail : kaboredonatien74@yahoo.fr
}

\begin{abstract}
\begin{tabular}{lll}
\hline Received: 20-04-2021 & Accepted: 24-08-2021 & Published: 31-08-2021 \\
\hline
\end{tabular}
\section{RESUME}

Le glutamate industriel (glutamate monosodique) représente à l'heure actuelle l'un des exhausteurs de goût les plus produits et consommés dans le monde. Cependant face à la menace de cet additif de synthèse sur la santé des consommateurs, des exhausteurs de goût d'origines naturelles sont de plus en plus recherchés dans notre alimentation. L'objectif de la présente étude est de produire des exhausteurs de goût d'origine naturelle à partir des coproduits issus de la production du Kilishi tels que les os, les parures de viande, des épices et ingrédients. Dans cette étude deux formulations de fonds de sauce concentrés de types Kilishi et deux formulations de fonds de sauce séchés ont été produits. Les qualités microbiologiques, nutritionnelles et sensorielles des fonds ont été évalués. Les résultats des analyses ont montré que les fonds de sauces concentrés de type Kilishi étaient de bonnes qualités nutritionnelles, microbiologiques, sensorielles, se conservaient bien à température ambiante et amélioreraient les goûts des saucisses. Par contre, les résultats des analyses microbiologiques ont montré que les fonds de sauces séchés n'étaient pas de bonne qualité microbiologique. La présente étude est une contribution à la diversification des exhausteurs de goût d'origine naturelle.
\end{abstract}

(C) 2021 International Formulae Group. All rights reserved.

Mots clés : Fonds de sauce, qualités physicochimique, microbiologique, sensorielle.

\section{Development of sauce base technology using co-products from the Kilishi production}

\begin{abstract}
Industrial glutamate is currently one of the most widely produced and consumed flavor enhancers in the world. But faced with the threat of this synthetic additive on health of consumers, flavor enhancers of natural origin are increasingly sought after in our food. The aim of the present study was to produce non-synthetic broths
\end{abstract}


used as flavor enhancers (sauces bases) from the co-products from Kilishi production such as bones, meat trimmings, spices and ingredients. In this study two formulations of concentrated sauce bases and dried sauces bases Kilishi -types were produced. The microbiological, nutritional and sensory qualities of different formulations of sauces bases were evaluated according to the respective standards methods. The results showed that the concentrated sauce bases of the Kilishi type were of good nutritional, microbiological and sensory qualities and kept well at room temperature. However, the results from microbiological analyzes showed that the dried sauce bases Kilishi- types were not of good microbiological quality. This study is a contribution to the diversification of natural flavor enhancers.

(C) 2021 International Formulae Group. All rights reserved.

Keywords: Sauce base, physicochemical, microbiological, sensory quality.

\section{INTRODUCTION}

Le glutamate représente à l'heure actuelle l'un des exhausteurs de goût le plus produit au monde (Boutry., 2010). Mais la consommation du glutamate sous sa forme libre (glutamate monosodique) poserait un problème de santé. En effet, la consommation à forte dose du glutamate industriel peut occasionner des migraines, des céphalées, des bouffées de chaleur, des paresthésies et les fourmillements (Deppenweiler, 2014). Des effets plus grave ont été décrits avec un lien potentiel entre glutamate et la destruction neuronale, l'autisme, la prise de poids voire l'obésité ou le diabète de type II, la fibromyalgie ou encore la dégénérescence rétinienne. Le glutamate aurait également un rôle physiopathologique dans le processus de cancérogénèse, dans l'épilepsie ou dans le développement de certaines maladies neurodégénératives comme la maladie d'Alzheimer, la maladie de Parkinson ou la sclérose en plaque. Ces effets ont cependant été relativement peu étudiés chez l'Homme puisque la majorité des études sont réalisées sur des animaux comme les souris et à de très fortes doses, largement supérieures aux doses nutritionnelles en glutamate libre. Des études supplémentaires sont nécessaires avant de pouvoir conclure aux mêmes symptômes chez l'homme (Boutry, 2010). Cependant le glutamate naturel contenu dans les aliments est majoritairement absorbé et toléré par l'organisme (Deppenweiler, 2014). Au vu des différentes données, il serait nécessaire de trouver des exhausteurs de goût d'origine naturelle comme les fonds de sauces. En effet, les fonds de sauces sont des préparations liquides utilisés comme exhausteurs de goûts et sont obtenus après une longue cuisson (trois à cinq heures) de viandes, d'os, de parures, d'arêtes de diverses origines (Poulain et al., 2017). La présente étude s'inscrit dans le sens de valoriser les coproduits issues de production du Kilishi tels que les os de bovins, les parures de viandes et les épices et ingrédients locaux issus de la production du Kilishi en exhausteur de goût d'origine naturelle (fonds de sauce).

\section{MATERIEL ET METHODES \\ Méthode de production et échantillonnage des fonds de sauces}

Plusieurs essais ont été effectués en vue de proposer des diagrammes standardisés pour les fonds de sauces concentrés et séchés. Deux formulations d'épices et ingrédients de type Kilishi ont été retenues suite à des essaies de la mise au point de la technologie de production des saucisses en utilisant les épices et ingrédients du Kilishi (Figure 1) (Derra, 2021). Dans ces travaux, les six formulations qui ont servi à la production des saucisses ont été réalisées sur la base des données d'étude qui a permis de retenir treize formulations proposées pour l'élaboration de la sauce d'enrobage des lanières de viandes séchées pour la production du Kilishi (Igene et al.,1990; Musonge et 
Njolai, 1993 ; Badau et al., 1997 ; Kalilou et al.,1998; Ogunsola et Omojola, 2008; Chukwu et Imodiboh, 2009; Mgbemere et al., 2011 ; Isa et al., 2012 ; Olusola et al., 2012 ; Jega et al., 2013 ; Bello et al., 2015 ; Iheagwara et Okonkwo, 2016) . Ces formulations ont permis de distinguer trois types d'épices et d'ingrédients constituants la sauce d'enrobage : les matières premières de bases, les épices et ingrédients qui ont été qualifiés d'obligatoires étaient présents dans toutes les formulations proposées par les auteurs et ceux qui n'étaient pas présents dans toutes les formulations étaient qualifiés de facultatifs. Également les valeurs minimales, moyennes, maximales des épices et ingrédients pour l'ensemble des treize formulations étudiées ont été déterminées. Sur cette base, une dernière étape de traitement de données a permis de générer cinq (05) formulations. La formulation témoin utilisée est celle proposée par Igene et Farouk (1990). Cette formulation témoin contenait un exhausteur de goût de type glutamate monosodique. La composition des six (6) formulations est mentionnée dans le Tableau1. Les résultats de ces travaux ont montré que les formulations F5 et témoin ont été parmi les six formules étudiés les meilleures formulations qui ont permis de produire les saucisses les plus appréciés par les dégustateurs Nous avons ainsi obtenue pour cette étude, les formulations 1 et 2 en remplaçant l'eau et l'exhausteur de goût de type glutamate monosodique présente dans les deux formulations par le fond de sauce nature. Trois (03) essais de production des fonds de sauces concentrés et séchés ont été réalisés selon les deux formulations 1 et 2 (Tableau 2). Trois (03) échantillons de fonds de sauces de chaque type ont été prélevés en raison de $500 \mathrm{~g}$ par échantillon et conservés au réfrigérateur à $4{ }^{\circ} \mathrm{C}$ pour les analyses physicochimiques, microbiologiques et sensorielles (Tableau 3).

\section{Méthode de production et échantillonnage des saucisses}

En vue d'évaluer la qualité sensorielle des fonds de sauces étudiés, 5\% des formulations 1 et 2 des fonds de sauces produits qui s'étaient avérés de bonne qualité microbiologique et nutritionnelle ont été utilisés comme exhausteur de goût pour produire deux formulations de saucisses. Ces saucisses ont été comparées aux saucisses qui contenait les mêmes quantité d'épices et ingrédients que les saucisses issues de la formulation 5 (Derra, 2021) mais, elle ne contenait pas de fond de sauce. Les trois formulations saucisses ont été produites selon le diagramme mentionné dans la Figure1. Trois (03) essais de productions de saucisses ont été réalisées et trois (03) échantillons de saucisses de chaque type ont été prélevés en raison de $500 \mathrm{~g}$ par échantillon et conservés au réfrigérateur à $4{ }^{\circ} \mathrm{C}$ pour les analyses physicochimiques, microbiologiques et sensorielles (Tableau 3).

\section{Analyses physico-chimiques}

L'analyse physico-chimique a concerné les paramètres tels que le $\mathrm{pH}$, l'humidité, les cendres, les protéines, les matières grasses, les glucides, l'énergie et l'humidité du produit dégraissé (HPD). Le pH a été mesuré à $25^{\circ} \mathrm{C}$ à l'aide du pH-mètre CONSORT P901 préalablement calibré avec des solutions tampons $\mathrm{pH} 4$ et $\mathrm{pH}$ 7. Cinq gramme (05g) de l'échantillon broyé ont été prélevés et additionnés à de l'eau distillée de manière à obtenir un volume total de $20 \mathrm{ml}$. Après homogénéisation, le pH affiché sur l'écran du pH-mètre a été relevé (Nout et al., 1998). La teneur en eau est déterminée par différence de pesée d'une prise d'essai avant et après passage à l'étuve selon la norme ISO 665 (2000). Les teneurs en Humidité du Produit Dégraissé (HPD) ont été déterminées suivant la formule décrite dans le code d'usage de la charcuterie et de la salaison et de conserve de viande (2016) : 


$$
\text { HPD }=\frac{100 \times \% \mathrm{H}}{100-\% \text { lipide }}
$$

HPD = l'humidité du produit dégraissé $; \% \mathrm{H}$ $(\mathrm{g} / \mathrm{g})=$ pourcentage d'humidité

La cendre ou matière minérale est le produit résultant de l'incinération de la matière organique. L'incinération est réalisée de façon à obtenir la totalité des cations sous forme de carbonates et autres sels minéraux anhydres (AOAC, 1990). Après l'incinération le poids de la matière minérale résultante est obtenu par pesée. La méthode Kjeldahl selon la norme AFNOR V03-050 (1970) a permis de déterminer la teneur en protéine. Sous l'action oxydante de l'acide sulfurique concentré bouillant en présence d'un catalyseur, l'azote organique de l'échantillon à analyser est transformé en azote minérale sous forme ammoniacale $\left(\mathrm{NH}_{4}\right)_{2} \mathrm{SO}_{4}$. L'ammoniac est distillé puis titré par acidimétrie en présence d'un réactif coloré (acide borique) après déplacement par la soude. La teneur en protéines totales est calculée en multipliant la quantité d'azote par un facteur de conversion $(6,25)$ soit $16 \%$ d'azote dans les protéines. La teneur en matières grasses a été déterminée par la méthode de type Soxhlet décrite selon la norme ISO 659 (1998), le principe de sa détermination est basé sur le fait que les lipides sont solubles dans les solvants organiques apolaires (l'hexane, l'éther à chaud). La valeur énergétique a été calculée en utilisant les coefficients d'Atwater et Benedict (1899) et les glucides totaux ont été déterminés par la méthode de différence :

$$
G(\%)=100-(\% H+\% C+\% M G+\% P)
$$

Avec :

$\% \mathrm{H}$ : teneur en d'eau ; \%C : teneur en cendre ; $\% \mathrm{P}$ : teneur protéines; \%MG : teneur en matière grasse ; $\% \mathrm{G}$ : Teneur en glucides.

L'énergie en $\mathrm{kJ}$ des différents produits a été obtenue par la méthode suivante :

$\% \mathrm{E}=(\% \mathrm{P} \times 17 \mathrm{~kJ})+(\% \mathrm{MG} \times 38 \mathrm{~kJ})+$ $(\% \mathrm{G} \times 17 \mathrm{~kJ})$
$\% \mathrm{P}:$ teneur protéines ; \% $\mathrm{MG}:$ teneur en matière grasse ; \%G : Teneur en glucides.

\section{Analyses microbiologiques \\ Préparation du diluant, des milieux de culture et des réactifs}

Les instructions du fabricant pour la préparation et le mode d'emploi de chaque milieu de culture et de chaque réactif ont été respectueusement appliquées.

\section{Préparation du diluant}

Pour préparer le diluant, 8,5 g de poudre déshydratée de chlorure de sodium $(\mathrm{NaCl})$ et 1 $\mathrm{g}$ de peptone sont dissous dans $1 \mathrm{~L}$ d'eau distillée. La solution est bien homogénéisée, puis le $\mathrm{pH}$ est mesuré et ajusté $(\mathrm{pH}=7 \pm 0,2)$. Le diluant obtenu est distribué dans des tubes à essai $(9 \mathrm{~mL} /$ tube) pour les dilutions décimales et en flacons $(90 \mathrm{~mL} /$ flacon $)$ pour la préparation des suspensions mères. Les tubes et les flacons contenant le diluant sont ensuite stérilisés à $121{ }^{\circ} \mathrm{C}$ pendant 15 minutes à l'autoclave.

Préparation de la suspension mère et des dilutions décimales pour l'ensemencement

La préparation des échantillons, de la suspension mère et des dilutions décimales est effectuée selon la norme internationale ISO 6887-1(1999). Les échantillons sont analysés immédiatement après le prélèvement. Dix (10) $\mathrm{g}$ d'échantillon sont pesés dans un sachet stomacher stérile dans lequel sont ajoutées 90 $\mathrm{mL}$ d'eau peptonée stérile. L'ensemble est homogénéisé au stomacher pendant 2 minutes. À partir de cette suspension mère, une série de dilutions décimales successives est réalisée de la manière suivante : $1 \mathrm{~mL}$ de solution est prélevé à l'aide d'une pipette et introduit dans un tube à essai contenant $9 \mathrm{~mL}$ d'eau peptonée stérile à la température ambiante ; un (1) $\mathrm{mL}$ est de nouveau prélevé de cette dernière solution et introduit dans le tube suivant contenant la même quantité d'eau peptonée. La dilution est ainsi faite jusqu'à la plus forte dilution désirée. 


\section{Ensemencement et incubation}

La méthode d'inoculation dans la masse a été utilisée pour l'ensemencement. Pour ce faire, $1 \mathrm{~mL}$ de chaque dilution décimale retenue a été prélevé aseptiquement et introduit dans une boîte de Pétri stérile. Ensuite environ 15-20 mL de milieu de culture stérilisé et maintenu en surfusion ont été ajoutés sur le prélèvement. Le tout a été mélangé en effectuant des rotations avec les boîtes couvertes. Les boîtes ont été laissées pour solidification pendant 5-10 minutes, avant d'être incubées aux températures et durées requises pour chaque microorganisme recherché. Les conditions d'incubation sont indiquées dans le Tableau 4.

\section{Comptage et expression des résultats :}

Après l'incubation, les colonies ont été comptées. Les boîtes de deux dilutions successives entre 4 et 300 colonies (flore totale) et entre 4 et 150 colonies (Coliformes totaux, Staphylococcus aureus, Levures et Moisissures) ont été retenues et le nombre de microorganismes $(\mathrm{N})$ a été calculé selon la formule suivante :

$$
\mathrm{N}=\frac{\Sigma C}{(1,1) v d}
$$

$\mathrm{N}$ : nombre total de germes, exprimé en UFC/g; $\Sigma \mathrm{C}$ : somme des colonies des boîtes de deux dilutions successives; $\mathrm{d}$ : dilution à partir de laquelle les premiers dénombrements sont obtenus ; v : volume de l'inoculumn.

- dans les cas où le nombre de colonies, est compris entre 01 et 03 dans les boites de dilution $10^{-1}$, les résultats sont exprimés comme suit : Moins de 40 microorganismes /g

- s'il n'y a aucune colonie dans les boîtes de dilution $10^{-1}$, le résultat est de moins de 10 microorganismes /g.

\section{Analyses sensorielles}

Les tests sensoriels ont consisté à des épreuves hédoniques. Un panel de 24 dégustateurs qui ont l'habitude de consommer les saucisses ont été retenus pour la réalisation de l'évaluation du test hédonique. Pour ce faire, les échantillons ont été présentés de façon simultanée et le sujet a exprimé son avis sur le goût des échantillons. L'analyse des données a été faite selon la méthode de Watts et al. (1991). Les descripteurs ont été convertis en notation numérique allant de 1 à 5 ou 5 correspond à «très agréable », 4 à «agréable », 3 à ni agréable ni désagréable, 2 à « désagréable » et 1 à «très désagréable ».

\section{Etude de la stabilité}

L'étude de la stabilité a consisté à garder les fonds de sauce concentrés de type Kilishi pendant 04 mois à la température ambiante et à prélever chaque mois des échantillons pour des analyses microbiologiques.

\section{Traitement des données d'analyses}

Les données d'analyses sensorielles, physicochimiques et microbiologiques ont été traitées à l'aide des logiciels Microsoft Excel/2013 et XLStat 2016. Le traitement a permis de caractériser les données quantitatives, essentiellement par leur moyenne, comme paramètre de tendance centrale. Les variables qualitatives des données d'analyses physicochimiques sont présentées avec leurs écarts types. Pour les paramètres physicochimiques, une analyse de la variance ANOVA à partir du test de Tukey, dont le risque d'erreur $(\alpha)$ était de $5 \%$ a été effectuée. L'évaluation sensorielle a été effectuée avec le test de Friedman dont le risque d'erreur $(\alpha)$ était de $5 \%$. 
Viande de bœuf

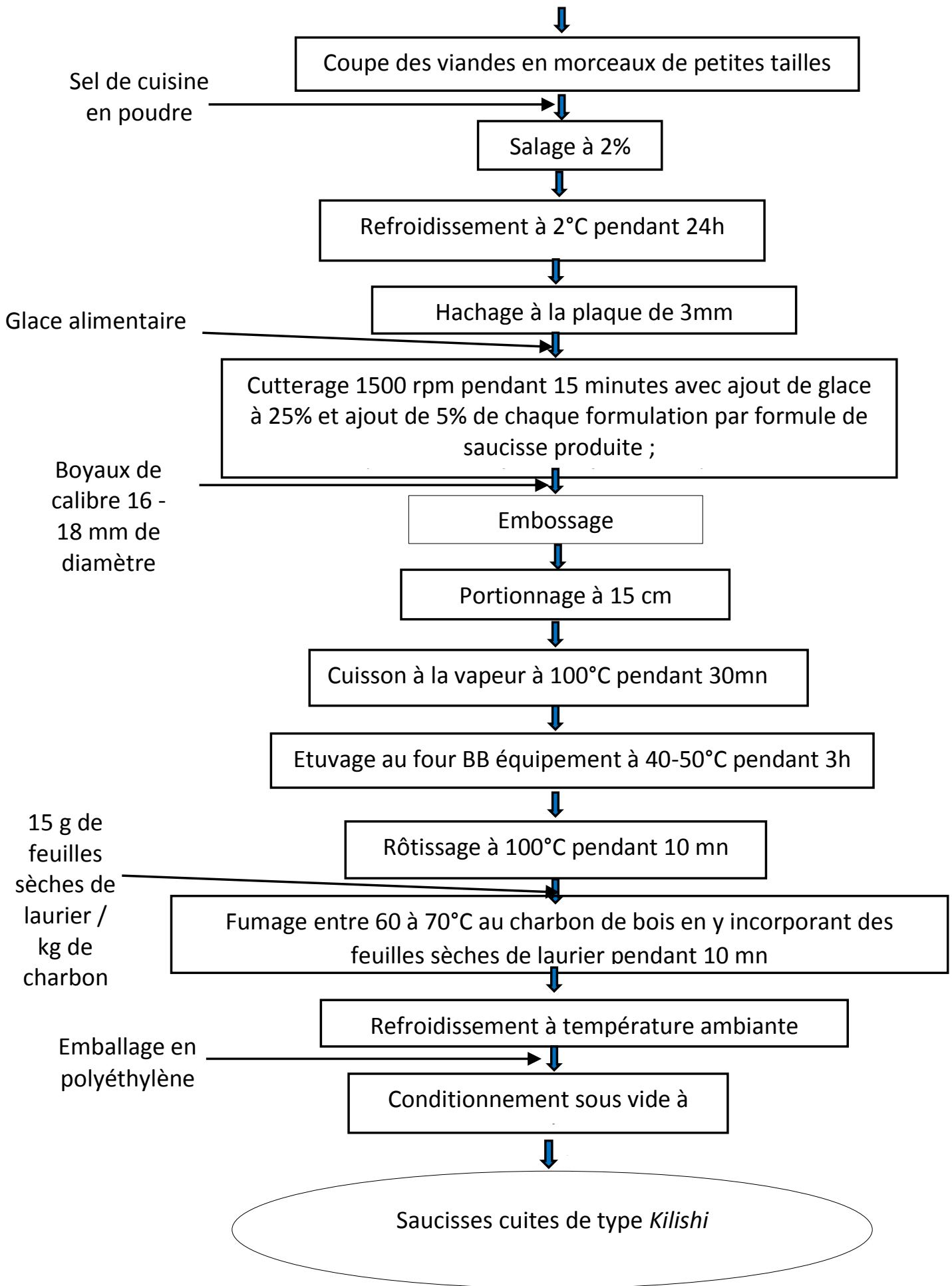

Figure 1 : Diagramme de production des saucisses cuites de type Kilishi (Derra, 2021). 
Tableau 1 : Compositions des six formulations des épices et ingrédients.

\begin{tabular}{|c|c|c|c|c|c|c|c|}
\hline \multicolumn{2}{|c|}{$\begin{array}{l}\text { Compositions des } \\
\text { formulations }\end{array}$} & F1 & F2 & F3 & F4 & F5 & FT \\
\hline \multirow[b]{2}{*}{$\begin{array}{l}\text { Matières } \\
\text { premières } \\
(\mathrm{g})\end{array}$} & Eau & 52,92 & 41,00 & 40,28 & 39,93 & 30,00 & 35,45 \\
\hline & $\begin{array}{l}\text { Tourteaux } \\
\text { d'arachide }\end{array}$ & 45,00 & 36,40 & 35,00 & 35,00 & 22,29 & 35,20 \\
\hline \multirow{4}{*}{$\begin{array}{l}\text { Epices et } \\
\text { ingrédients } \\
\text { obligatoires }\end{array}$} & Oignons & 0,55 & 6,23 & 11,73 & 0,55 & 11,73 & 11,73 \\
\hline & Piments & 0,53 & 1,66 & 4,69 & 0,53 & 4,69 & 4,69 \\
\hline & Gingembres & 0,60 & 2,44 & 3,80 & 0,60 & 3,80 & 2,35 \\
\hline & Sel & 0,40 & 2,02 & 4,50 & 0,4 & 4,50 & 1,88 \\
\hline \multirow{9}{*}{$\begin{array}{l}\text { Epices et } \\
\text { ingrédients } \\
\text { facultatifs } \\
\text { (g) }\end{array}$} & $\begin{array}{l}\text { Poivre de } \\
\text { Guinée }\end{array}$ & 0 & 1,49 & 0 & 3,40 & 3,40 & 1,17 \\
\hline & Poivre noir & 0 & 1,84 & 0 & 4,69 & 4,69 & 4,69 \\
\hline & $\begin{array}{l}\text { Clou de } \\
\text { girofle }\end{array}$ & 0 & 0,88 & 0 & 2,60 & 2,60 & 0 \\
\hline & $\begin{array}{l}\text { Fond blanc } \\
\text { de bœuf }\end{array}$ & 0 & 3,08 & 0 & 5,80 & 5,80 & 0 \\
\hline & $\begin{array}{l}\text { Exhausteur } \\
\text { de goût de type } \\
\text { MSG }\end{array}$ & 0 & 0 & 0 & 0 & 0 & 0,14 \\
\hline & $\begin{array}{l}\text { Colorant } \\
\text { rouge }\end{array}$ & 0 & 0,41 & 0 & 2,00 & 2,00 & 0,23 \\
\hline & Sucre & 0 & 2,00 & 0 & 3,50 & 3,50 & 1,88 \\
\hline & $\begin{array}{l}\text { Fausse noix } \\
\text { de muscade }\end{array}$ & 0 & 0,55 & 0 & 1,00 & 1,00 & 0,59 \\
\hline & Total en $\mathrm{g}$ des formulations & 100 & 100 & 100 & 100 & 100 & 100 \\
\hline
\end{tabular}

Légende : F= Formulation ; T= Témoin

Source : Derra (2021)

Tableau 2 : Formulation des fonds de sauces.

\begin{tabular}{|c|c|c|c|}
\hline \multicolumn{2}{|c|}{ Compositions des formulations } & \multicolumn{2}{|c|}{ Formulations } \\
\hline & & F1 & F2 \\
\hline \multirow{3}{*}{$\begin{array}{l}\text { Matières } \\
\text { premières de } \\
\text { bases (g) }\end{array}$} & Eau & 0 & 0 \\
\hline & $\begin{array}{l}\text { Fonds de sauce } \\
\text { nature }\end{array}$ & 35,80 & $\begin{array}{l}35,59 \\
0\end{array}$ \\
\hline & $\begin{array}{l}\text { Tourteaux } \\
\text { d'arachide }\end{array}$ & 22,29 & 35,20 \\
\hline \multirow{4}{*}{$\begin{array}{l}\text { Ingrédients } \\
\text { obligatoires } \\
\text { (g) }\end{array}$} & Oignons & 11,73 & 11,73 \\
\hline & Piments & 4,69 & 4,69 \\
\hline & Gingembres & 3,80 & 2,35 \\
\hline & Sel & 4,5 & 1,88 \\
\hline
\end{tabular}




\begin{tabular}{lllc}
\hline \multirow{2}{*}{$\begin{array}{l}\text { Ingrédients } \\
\text { facultatifs }(\mathrm{g})\end{array}$} & Poivre noir & 4,69 & 4,69 \\
\cline { 2 - 3 } & $\begin{array}{l}\text { Poivre de } \\
\text { Guinée }\end{array}$ & 3,40 & 1,17 \\
\cline { 2 - 3 } & Clou de girofle & 2,60 & 0 \\
\cline { 2 - 3 } & Colorant rouge & 2,00 & 0,23 \\
\cline { 2 - 3 } & Sucre & 3,50 \\
\cline { 2 - 3 } & $\begin{array}{l}\text { Fausse noix de } \\
\text { muscade }\end{array}$ & 1,00 & 0,59 \\
\hline
\end{tabular}

Légende : F : Formulation

Tableau 3 : Prélèvement des échantillons par types d'analyses.

\begin{tabular}{|c|c|c|}
\hline Echantillons & Types d'analyses & $\begin{array}{l}\text { Nombre } \\
\text { d'échantillon } \\
\text { par types de } \\
\text { produits }\end{array}$ \\
\hline
\end{tabular}

Fond de sauce Nature

Fond de sauce concentré types Kilishi formulation 1 (FSCTK1)

Fond de sauce concentré types Kilishi formulation 2 (FSCTK2)

Fond de sauce séché type Kilishi formulation 1 Analyse microbiologique: Flore Mésophile Aérobie Totale (FMAT), Coliforme totaux, $S$. aureus, B. cereus, levures et moisissures

Analyse physicochimique: Teneur en eau, cendres, matières grasses, protéines, glucides totaux. (FSSTK1)

Fond de sauce séché type Kilishi formulation 2 (FSSTK2)

\begin{tabular}{lll}
\hline $\begin{array}{l}\text { Saucisses cuites à base des } \\
\text { fonds de sauces concentrés } \\
\text { de type Kilishi de la } \\
\text { formulation } 1 \text { (SFSCTK1) }\end{array}$ & $\begin{array}{l}\text { Analyse microbiologique : Flore } \\
\text { Mésophile Aérobie Totale (FMAT), } \\
\text { Coliforme totaux, S. aureus, B. } \\
\text { cereus, levures et moisissures }\end{array}$ & 03 \\
$\begin{array}{l}\text { fonds de sauces concentrés } \\
\text { Analyse physicochimique : Teneur } \\
\text { en eau, cendres, matières grasses, }\end{array}$ & 03 \\
$\begin{array}{l}\text { formulation 2 (SFSCTK2) } \\
\text { sauce (témoin) }\end{array}$ & $\begin{array}{l}\text { protéines, glucides totaux, HPD } \\
\text { Analyse sensorielle : Test hédonique }\end{array}$ & 03 \\
\hline Total échantillon & Total analyse & 24 \\
\hline
\end{tabular}


Tableau 4 : Milieux de cultures, méthodes et conditions d'incubation.

\begin{tabular}{lccc}
\hline $\begin{array}{l}\text { Microorganismes } \\
\text { Recherchés }\end{array}$ & $\begin{array}{c}\text { Références de la } \\
\text { méthode }\end{array}$ & $\begin{array}{c}\text { Milieux de cultures } \\
\text { utilisés }\end{array}$ & $\begin{array}{c}\text { Température/temps } \\
\text { d'incubation }\end{array}$ \\
\hline $\begin{array}{l}\text { Flore mésophile } \\
\text { aérobie totale }\end{array}$ & NF EN ISO 4833 (2003) & Plate Count Agar (PCA) & $30{ }^{\circ} \mathrm{C}$ pendant $48-72$ heures \\
\hline Coliformes totaux & ISO 21528-2 (2004) & $\begin{array}{c}\text { Violet Red Bile Glucose } \\
\text { Agar (VRBL) }\end{array}$ & $37{ }^{\circ} \mathrm{C}$ pendant 24 heures \\
\hline $\begin{array}{l}\text { Staphylococcus } \\
\text { aureus }\end{array}$ & ISO 4832-2 (2006) & $\begin{array}{c}\text { BAIRD PARKER (BP) } \\
37{ }^{\circ} \mathrm{C} \text { pendant } 24 \text { heures }\end{array}$ \\
\hline $\begin{array}{l}\text { Bacillus cereus } \\
\text { Levures et }\end{array}$ & ISO 7932(E) 1993 & $\begin{array}{c}\text { Brillance Bacillus } \\
\text { cereus (BBC) }\end{array}$ & $30{ }^{\circ} \mathrm{C}$ pendant $24 \mathrm{~h}$ \\
moisissures & NF ISO 7954. (1988) & $\begin{array}{c}\text { Sabouraud (SAB) } \\
\text { A } 30^{\circ} \mathrm{C} \text { pendant } 72 \mathrm{~h}\end{array}$ \\
\hline
\end{tabular}

\section{RESULTATS}

\section{Technologies des fonds de sauces concentrés et séchées de type Kilishi}

Les fonds de sauces ont été produits à partir des os de bovins suivant les étapes suivantes: concassage des os, mouillage et ébullition, refroidissement, addition d'eau, cuisson légère et régulière, filtration puis obtention d'un fond de sauce nature. Le fond de sauce nature a été utilisé pour fabriquer le fond de sauce concentré par ajout des épices et ingrédients de type kilishi, le mélange est mixé et conditionné dans les boîtes en verre puis refermé à l'aide d'un couvercle métallique, ces boîtes ont été par la suite stérilisées. La technologie de production des fonds de sauces concentrés est décrite dans la Figure 2.

Quant à la technologie des fonds de sauce séchés, les épices et ingrédients sont ajoutés au fonds de sauces nature puis mixés et conditionnés dans des petits moules pour être séchés, rôtis et fumés dans le four à gaz. La technologie de production des fonds de sauces séchés est décrite dans la Figure 3.

\section{Caractéristiques physicochimiques des fonds de sauces étudiés}

Les caractéristiques physicochimiques des fonds de sauces natures, concentrés et séchés sont présentées dans le Tableau 5. Les valeurs des teneurs en eau, en cendres, en protéines, en matières grasses, en glucides par rapport à la matière sèche ainsi que les valeurs énergétiques des fonds de sauces étudiés ont respectivement varié de 11,72 à $98,81 \% ; 8,81$ à $14,56 \% ; 20,00$ à $69,72 \% ; 12,47$ à $25,01 \%$; 0,96 à $52 \% ; 327,70$ à $1715 \mathrm{~kJ} / 100 \mathrm{~g}$ et le $\mathrm{pH}$ 5,09 à 6,60. L'analyse de la variance ANOVA $(\mathrm{p}<0,05)$ indique qu'il y a une différence significative entre les valeurs des teneurs en eau, en matières grasses en protéines, en glucides, en énergie et les $\mathrm{pH}$ des fonds sauces étudiés.

\section{Caractéristiques physicochimiques des saucisses produites à partir des fonds de sauces}

Les caractéristiques physicochimiques des saucisses avaient des teneurs en eau, cendres, matières grasses, glucides, protéines ainsi que des valeurs énergétiques, du pH et du 
HPD qui ont varié respectivement de 54,05 à $64,77 \% \mathrm{mh} ; 2,25$ à $2,35 \% \mathrm{mh} ; 3,38$ à $9,78 \%$ $\mathrm{mh} ; 1,49$ à $3,96 \% \mathrm{mh} ; 27,50$ à $32,67 \% \mathrm{mh}$; 622,32 à $884,19 \mathrm{~kJ} / 100 \mathrm{~g} ; 5,09$ à 6,60 et de 58,10 à 67,03\% mh (Tableau 6). L'analyse de la variance ANOVA $(p<0,05)$ indique qu'il y a une différence significative entre les valeurs des teneurs en eau, en cendre, en matières grasses, en glucides, protéines, en énergie, les pH et du HPD des saucisses étudiées.

\section{Caractéristiques microbiologiques des fonds de sauces étudiés}

Les résultats des analyses microbiologiques effectuées sur les fonds de sauces sont mentionnés dans le Tableau 7. La charge en UFC/g de la flore mésophile aérobie totale a varié de moins de 10 à $6,9.10^{6}$, celle des B. cereus a varié de moins de 10 à $6,9.10^{5}$ et des S. aureus de moins 10 à $4,910^{1}$. Quant aux charges en UFC/g des coliformes totaux, des levures et moisissures, elles étaient de moins de 10 chacune. Les résultats des analyses microbiologiques effectuées sur saucisses sont consignés dans le Tableau 8. La charge en UFC/g de la flore mésophile aérobie totale a varié de moins de 10 à $7,1.10^{2}$ dans les trois formulations de saucisses. Quant aux charges en UFC/g des coliformes totaux, des levures et moisissures, des $B$. cereus et $S$. aureus, elles étaient de moins de 10 chacune.
Etude de la stabilité des fonds de sauces concentrés de type Kilishi

Cette étude visait à déterminer la stabilité des fonds concentrés pendant quatre (04) mois de conservation. Les résultats sont indiqués dans le Tableau 9 et montrent que la charge en ufc/g des microorganismes analysés (Flore totale, Coliformes totaux, levures et moisissures, B. cereus et $S$. aureus) n'a pas varié au cours du temps (t). Les échantillons analysés à $\mathrm{t}=0, \mathrm{t}=1$ mois, $\mathrm{t}=2$ mois, $\mathrm{t}=3$ mois, $\mathrm{t}=4$ mois avaient des charges en UFC/g identiques pour tous les microorganismes analysés et étaient de moins $10 \mathrm{UFC} / \mathrm{g}$ pour chaque paramètre microbiologique analysé.

\section{Caractéristiques sensorielles des saucisses étudiées}

Des saucisses ont été produites à partir des fonds de sauces concentrés TK1 et TK2 qui étaient de bonne qualité microbiologique. Les fonds de sauce concentrés ont été ajoutés à une proportion de $5 \%$ de la quantité de viande utilisée pour la production des deux formulations de saucisses et ont été comparé à une formulation de saucisses produites sans ajout de fonds de sauces Ces saucisses ont été toutes appréciées par le panel de dégustateurs de 24personnes. Les scores du test d'acceptabilité ont varié de 4,04 à 4,54. Ces scores se situent entre 4 et 5 : elles correspondent aux goûts agréable et très agréable. Les résultats du test d'acceptabilité suite à l'appréciation du panel de dégustateurs de 24 personnes sont représentés dans le Tableau 10. 


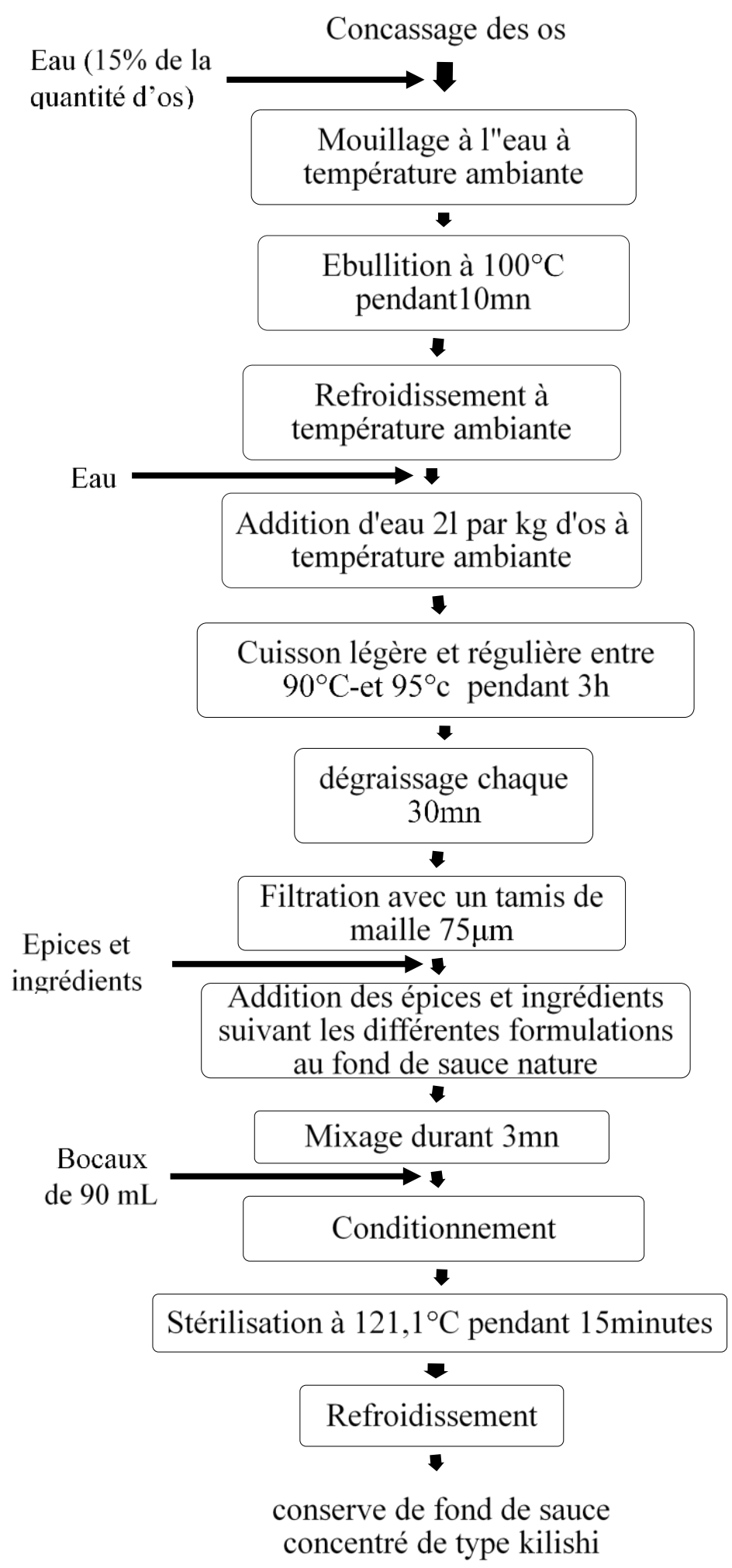

Figure 2 : Diagramme de production des fonds de sauces concentrés de type Kilishi. 


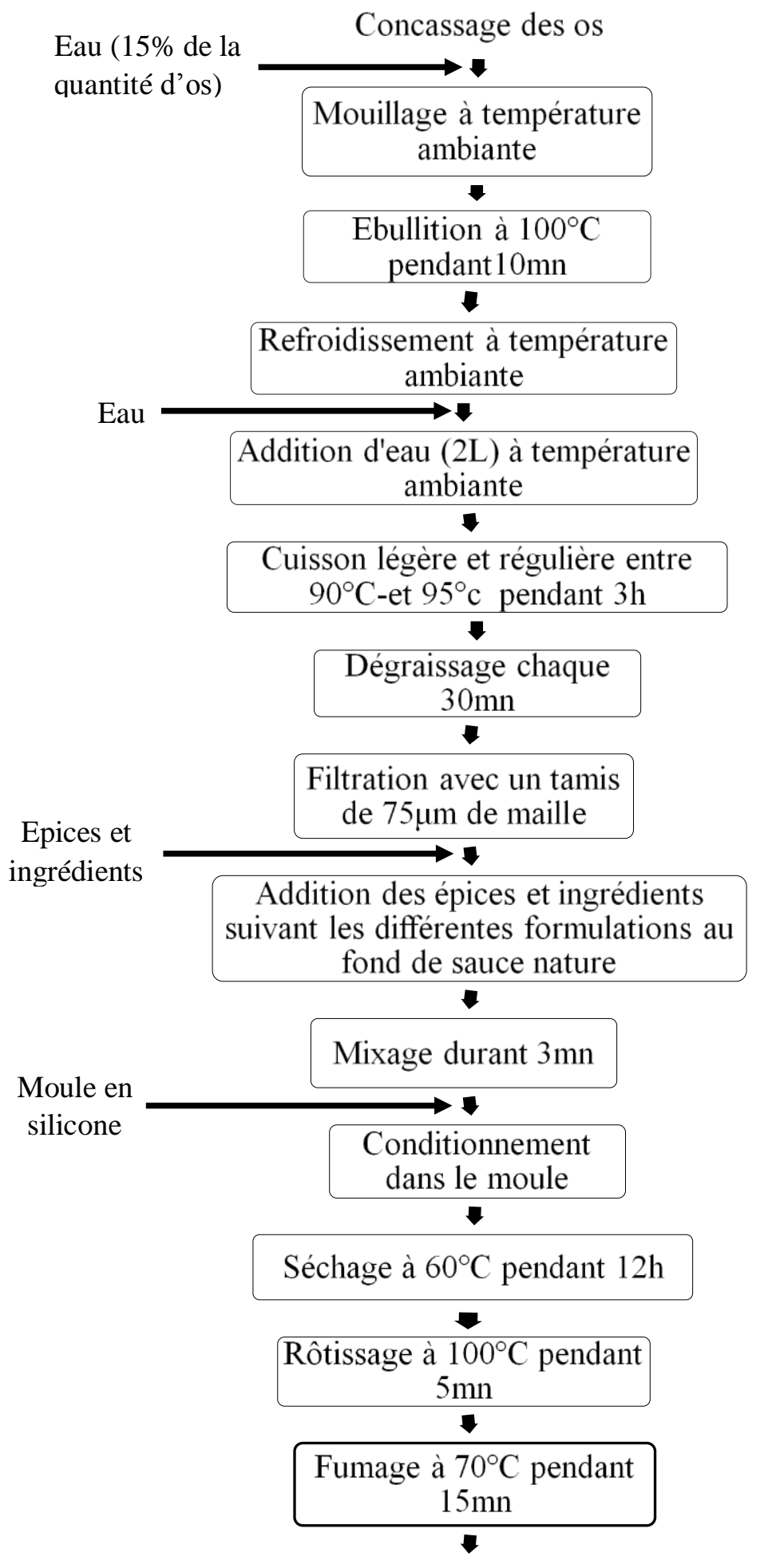

Fonds de sauces séché de type kilishi

Figure 3 : Diagramme de production des fonds de sauces séchés de type Kilishi. 
Tableau 5 : Caractéristiques physicochimiques des fonds de sauces.

\begin{tabular}{|c|c|c|c|c|c|c|c|}
\hline Echantillons & $\begin{array}{l}\text { Humidité } \\
\text { (\% ms) }\end{array}$ & $\begin{array}{l}\text { Cendres } \\
(\% \mathrm{~ms})\end{array}$ & $\begin{array}{l}\text { Protéines } \\
(\% \mathrm{~ms})\end{array}$ & $\begin{array}{l}\text { Matières } \\
\text { Grasses } \\
(\% \mathrm{~ms})\end{array}$ & $\begin{array}{l}\text { Glucides } \\
\text { (\% } \mathrm{ms})\end{array}$ & $\begin{array}{l}\text { Energie } \\
(\mathrm{kJ} / \mathbf{1 0 0 g})\end{array}$ & $\mathrm{pH}$ \\
\hline \multirow[t]{2}{*}{ Fonds de sauce Nature } & $98,81^{\mathrm{c}}$ & 9,32 & $69,72^{\mathrm{d}}$ & $20,00^{\mathrm{b}}$ & $0,96^{\mathrm{a}}$ & $327,70^{\mathrm{a}}$ & $6,60^{\mathrm{e}}$ \\
\hline & \pm 0 & $\pm 0,15$ & $\pm 3,54$ & $\pm 3,31$ & $\pm 0,48$ & $\pm 52,06$ & \pm 0 \\
\hline Fonds de sauce & $55,27^{\mathrm{b}}$ & $14,17^{\mathrm{c}}$ & $22,88^{\mathrm{ab}}$ & $19,15^{\mathrm{b}}$ & $43,80^{c}$ & $1050,20^{\mathrm{b}}$ & $5,62^{\mathrm{c}}$ \\
\hline Concentré TK1 & $\pm 0,14$ & $\pm 0,06$ & $\pm 0,55$ & $\pm 0,67$ & $\pm 0,99$ & $\pm 11,85$ & \pm 0 \\
\hline Fonds de sauce & $58,56^{\mathrm{b}}$ & $8,81^{\mathrm{a}}$ & $28,05^{\mathrm{bc}}$ & $25,01^{\mathrm{c}}$ & $38,12^{\mathrm{b}}$ & $1165,01^{\mathrm{c}}$ & $6,04^{\mathrm{d}}$ \\
\hline Concentré TK2 & $\pm 0,16$ & $\pm 0,11$ & $\pm 0,11$ & $\pm 0,25$ & $\pm 0,09$ & $\pm 7,80$ & $\pm 0,01$ \\
\hline Fonds de Sauce & $12,73^{\mathrm{a}}$ & $14,56^{\mathrm{d}}$ & $20,00^{\mathrm{a}}$ & $12,47^{\mathrm{a}}$ & $52,97^{\mathrm{d}}$ & $1512,47^{\mathrm{d}}$ & $5,09^{\mathrm{a}}$ \\
\hline Séchés TK1 & $\pm 0,33$ & $\pm 0,18$ & $\pm 3,01$ & $\pm 0,55$ & $\pm 3,51$ & $\pm 17,83$ & $\pm 0,01$ \\
\hline Fonds de Sauce & $11,72^{\mathrm{a}}$ & $9,28^{\mathrm{b}}$ & $32,02^{\mathrm{c}}$ & $17,67^{\mathrm{b}}$ & $41,02^{\mathrm{bc}}$ & $1715,72^{\mathrm{e}}$ & $5,28^{\mathrm{b}}$ \\
\hline Séchés TK2 & $\pm 0,13$ & $\pm 0,01$ & $\pm 0,50$ & $\pm 0,73$ & $\pm 0,90$ & $\pm 16,37$ & \pm 0 \\
\hline
\end{tabular}

Les valeurs ayant des lettres en exposant sont significativement différentes $(\mathrm{P}<0,05)$

Légende : TK1 : Type Kilishi 1 ; TK2: Type Kilishi 2

Tableau 6 : Caractéristiques physicochimiques des saucisses produites à partir des fonds de sauces.

\begin{tabular}{|c|c|c|c|c|c|c|c|c|}
\hline Echantillons & $\begin{array}{l}\text { Humidité } \\
(\%)\end{array}$ & $\begin{array}{l}\text { HPD } \\
(\% \mathrm{mh})\end{array}$ & $\begin{array}{l}\text { Cendres } \\
(\% \mathrm{mh})\end{array}$ & $\begin{array}{l}\text { Protéines } \\
(\% \mathrm{mh})\end{array}$ & $\begin{array}{l}\text { Matières } \\
\text { Grasses } \\
(\% \text { mh })\end{array}$ & $\begin{array}{l}\text { Glucide } \\
\text { (\% mh) }\end{array}$ & $\begin{array}{l}\text { Energie } \\
(\mathrm{kJ} / \mathbf{1 0 0 g})\end{array}$ & PH \\
\hline $\begin{array}{l}\text { Saucisses issues } \\
\text { des fonds de } \\
\text { sauce Concentré } \\
\text { TK1 }\end{array}$ & $\begin{array}{l}54,05^{\text {a }} \\
\pm 0,29\end{array}$ & $\begin{array}{l}58,10^{\mathrm{a}} \\
\pm 0,10\end{array}$ & $\begin{array}{l}2,35^{\mathrm{a}} \\
\pm 0,05\end{array}$ & $\begin{array}{l}32,67^{b} \\
\pm 0,68\end{array}$ & $\begin{array}{l}6,97^{\mathrm{b}} \\
\pm 0,34\end{array}$ & $\begin{array}{l}3,96^{\mathrm{b}} \\
\pm 0,61\end{array}$ & $\begin{array}{l}877,52^{\mathrm{b}} \\
\pm 12,51\end{array}$ & $\begin{array}{l}5,64^{\mathrm{b}} \\
\pm 0,01\end{array}$ \\
\hline $\begin{array}{l}\text { Saucisses issues } \\
\text { des fonds de } \\
\text { sauce concentré } \\
\text { TK2 }\end{array}$ & $\begin{array}{l}64,77^{\mathrm{c}} \\
\pm 0,23\end{array}$ & $\begin{array}{l}67 \\
, 03^{c} \\
\pm 0,06\end{array}$ & $\begin{array}{l}2,32^{\mathrm{a}} \\
\pm 0,01\end{array}$ & & $\begin{array}{l}3,38^{\mathrm{a}} \\
\pm 0,37\end{array}$ & $\begin{array}{l}1,49^{\mathrm{a}} \\
\pm 0,26\end{array}$ & $\begin{array}{l}622,32^{\mathrm{a}} \\
\pm 12,11\end{array}$ & $\begin{array}{l}5,60^{\mathrm{a}} \\
\pm 0,02\end{array}$ \\
\hline $\begin{array}{l}\text { Saucisse sans } \\
\text { fonds de sauce } \\
\text { (Témoin) }\end{array}$ & $\begin{array}{l}57,34^{\mathrm{b}} \\
\pm 0,32\end{array}$ & $\begin{array}{l}63,56^{\mathrm{b}} \\
\pm 0,25\end{array}$ & $\begin{array}{l}2,25^{\mathrm{a}} \\
\pm 0,17\end{array}$ & $\begin{array}{l}27,50^{\mathrm{a}} \\
\pm 0,38\end{array}$ & $\begin{array}{l}9,79^{\mathrm{c}} \\
\pm 0,34\end{array}$ & $\begin{array}{l}3,11^{\mathrm{c}} \\
\pm 0,35\end{array}$ & $\begin{array}{l}884,19^{\mathrm{b}} \\
\pm 14,26\end{array}$ & $\begin{array}{l}5,61^{\mathrm{ab}} \\
\pm 0\end{array}$ \\
\hline
\end{tabular}

Les valeurs ayant des lettres en exposant sont significativement différentes $(\mathrm{P}<0,05)$

Légende : TK1 : Type Kilishi 1 ; TK2: Type Kilishi 2

Tableau 7 : Qualité microbiologique des fonds de sauces.

\begin{tabular}{llllll}
\hline Paramètres & $\begin{array}{l}\text { Flore Mésophile } \\
\text { Aérobique Totale } \\
\text { (ufc/g) }\end{array}$ & $\begin{array}{l}\text { Coliformes } \\
\text { Totaux } \\
\text { (ufc/g) }\end{array}$ & $\begin{array}{l}\text { Lévures et } \\
\text { Moisissures } \\
(\mathbf{u f c} / \mathbf{g})\end{array}$ & $\begin{array}{l}\text { Bacillus } \\
\text { cereus } \\
\text { (ufc/g) }\end{array}$ & $\begin{array}{l}\text { Staphylococcus } \\
\text { aureus } \\
\text { (ufc/g) }\end{array}$ \\
\hline $\begin{array}{l}\text { Fonds de sauce } \\
\text { nature }\end{array}$ & $2,4.10^{3}$ & $<10$ & $<10$ & $<10$ & $4,9.10^{1}$ \\
\hline
\end{tabular}




\begin{tabular}{llllll}
\hline $\begin{array}{l}\text { Fonds de sauce } \\
\text { concentré TK1 }\end{array}$ & $<10$ & $<10$ & $<10$ & $<10$ & $<10$ \\
\hline $\begin{array}{l}\text { Fonds de sauce } \\
\text { concentré TK2 }\end{array}$ & $<10$ & $<10$ & $<10$ & $<10$ & $<10$ \\
\hline $\begin{array}{l}\text { Fonds de } \\
\text { Séché TK1 }\end{array}$ & $8,4.10^{4}$ & $<10$ & $<10$ & $2,9.10^{4}$ & $<10$ \\
\hline $\begin{array}{l}\text { Fonds de } \\
\text { séché TK2 }\end{array}$ & $6,9.10^{6}$ & $<10$ & $<10$ & $6,9.10^{5}$ & $<10$ \\
\hline
\end{tabular}

Légende : TK1 : Type Kilishi 1; TK2: Type Kilishi 2, UFC : Unité Formant Colonie

Tableau 8 : Qualité microbiologique des saucisses.

\begin{tabular}{|c|c|c|c|c|c|}
\hline Echantillons & $\begin{array}{l}\text { Flore Mésophile } \\
\text { Anaérobique Totale } \\
\text { (ufc/g) }\end{array}$ & $\begin{array}{l}\text { Coliformes } \\
\text { Totaux } \\
\text { (ufc/g) }\end{array}$ & $\begin{array}{l}\text { Levures et } \\
\text { moisissures } \\
\text { (ufc/g) }\end{array}$ & $\begin{array}{l}\text { Bacillus } \\
\text { cereus (ufc/g) }\end{array}$ & $\begin{array}{l}\text { Staphylococcus } \\
\text { aureus } \\
\text { (ufc/g) }\end{array}$ \\
\hline $\begin{array}{l}\text { Saucisses issues } \\
\text { des fonds de } \\
\text { sauce Concentré } \\
\text { TK1 }\end{array}$ & $<10$ & $<10$ & $<10$ & $<10$ & $<10$ \\
\hline $\begin{array}{l}\text { Saucisses issues } \\
\text { des fonds de } \\
\text { sauce Concentré } \\
\text { TK2 }\end{array}$ & $<10$ & $<10$ & $<10$ & $<10$ & $<10$ \\
\hline $\begin{array}{l}\text { Saucisse sans } \\
\text { fonds de } \\
\text { sauces(Témoin) }\end{array}$ & $7,2.10^{2}$ & $<10$ & $<10$ & $<10$ & $<10$ \\
\hline
\end{tabular}

Légende : TK1 : Type Kilishi 1; TK2: Type Kilishi 2, UFC : Unité Formant Colonie

Tableau 9 : Evolution de la qualité microbiologique des fonds de sauce concentré en fonction du temps.

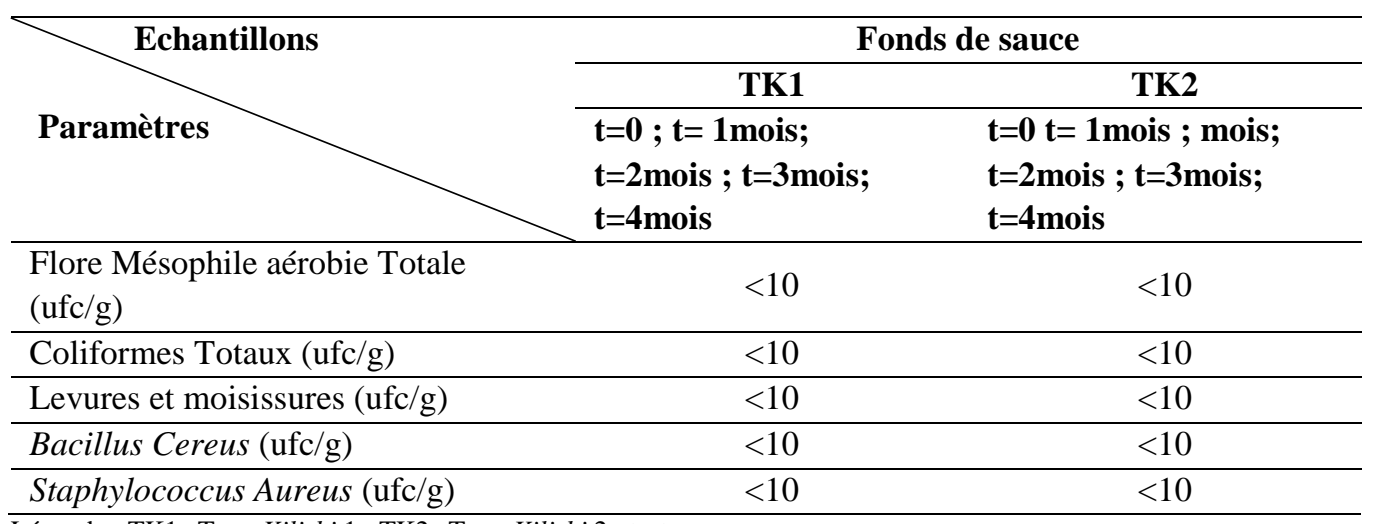

Légende : TK1 : Type Kilishi 1 ; TK2 : Type Kilishi 2 ; t= temps ;

UFC : Unité Formant Colonie 
Tableau 10 : Acceptabilité (test hédonique) des saucisses.

\begin{tabular}{llll}
\hline Echantillons & Goût (score) & Caractère & Classement \\
\hline $\begin{array}{l}\text { Saucisses issues des fonds de sauce } \\
\text { concentré TK2 }\end{array}$ & $4,54^{\mathrm{b}}$ & Agréable & $1^{\text {er }}$ \\
\hline $\begin{array}{l}\text { Saucisses issues des fonds de sauce } \\
\text { concentré K1 }\end{array}$ & $4,37^{\mathrm{ab}}$ & Agréable & $2^{\text {ème }}$ \\
\hline Saucisses sans fond de sauce & $4,04^{\mathrm{a}}$ & Agréable & $3^{\text {ème }}$ \\
\hline
\end{tabular}

Les valeurs ayant des lettres différentes en exposant sont significativement différentes $(\mathrm{P}<0,05)$

Légende : F : Formule, T : Témoin, TK1 : Type Kilishi 1 ; TK2: Type Kilishi 2

\section{DISCUSSION}

La technologie de fonds de sauces proposées dans cette étude a permis de valoriser les os de bovins issus de la production du Kilishi et d'utiliser les épices et ingrédients locaux (épices généralement utilisés dans la production du Kilishi). En effet, les différentes recettes de production de fonds de sauces existantes utilisent les épices importés (Polain et al., 2017). Les fonds de sauces produites dans notre étude à partir des os de bovin (riche en protéine) libèreraient au cours de la longue cuisson (3 à 4h) de l'acide glutamique (Kurihara., 2009). La présence de l'acide glutamique dans les fonds de sauces leurs conféreraient le rôle d'exhausteur de goût d'origine naturelle. En effet, le glutamate naturel contenu dans les aliments est majoritairement absorbé par l'intestin puis métabolisé dans les cellules épithéliales intestinales. De manière générale, les capacités métaboliques de l'intestin et $\mathrm{du}$ foie étant élevées, les concentrations postprandiales en glutamate restent stables. De nombreux récepteurs du glutamate ont été retrouvés dans les organes et tissus humains. Lorsque sa consommation excède les doses usuelles, les concentrations portales en glutamate peuvent augmenter ce qui conduit à une majoration de son métabolisme hépatique. Une ingestion abondante de glutamate monosodique (glutamate industrielle), qui est un sel sodique $\mathrm{du}$ glutamate, va stimuler tout autant les récepteurs du glutamate au niveau périphérique qu'au niveau central. Une consommation abondante de glutamate monosodique, peut conduire à un taux élevé de glutamate sanguin
(20 à 40 fois la normale) et ainsi constituer une menace pour certains organes (Deppenweiler, 2014). Les fonds de sauces produites pourraient donc remplacer les exhausteurs de goût de type glutamate monosodique qui sont très consommé dans le monde (Boutry, 2010).

D'une manière générale, l'analyse de la variance ANOVA $(\mathrm{P}<0,05)$ des résultats des analyses physicochimiques (Tableau 5) a montré qu'il y a une différence significative entre les valeurs des teneurs en eau, cendres, matières grasses, glucides ainsi que le $\mathrm{pH}$ et les valeurs énergétiques des fonds de sauces. Cette différence pourrait s'expliquer par la nature des fonds de sauces et leur composition qui diffèrent. En effet nos échantillons sont composés des fonds de sauces natures qui ne contiennent que de l'eau et des extraits d'os, de fonds de sauce concentré composé d'eau, d'extrait d'os et d'épices et ingrédients type Kilishi et de fonds de sauce séchés. En comparant les différentes valeurs des fonds de sauces étudiés avec ceux des os frais qui sont de $31,37 \%$ de lipide, $23,53 \%$ de protéine et $45,10 \%$ de sels minéraux (Linder, 1996). Nous constatons que les fonds de sauces étudiés avaient tous des valeurs en cendres $(8,81$ à $14,56 \%$ ) inférieures à celles des os qui est de $45,10 \%$. Cette faible concentration en sels minéraux de nos fonds par rapport à ceux des os pourrait s'expliquer par la méthode utilisé pour la production des fonds (longue cuisson des os de trois à quatre heures) qui n'a pas permis d'extraire tous les minéraux de l'os. Par contre les valeurs en protéines de nos fonds de sauces (20 à 69,72\%) étaient supérieurs à celles des os. Ces valeurs plus élevées en protéines 
des fonds de sauce étudiés pourraient s'expliquer par le faite que dans la production des fonds de sauce, les os utilisés contenaient également de la viande qui est très riche en protéine. Egalement en comparant les valeurs en glucides de nos fonds de sauce étudiés avec celles des os qui n'en contiennent pratique pas. Nous constatons que la teneur en glucides de nos fonds concentrés et séchés était très élevée $(38,12$ à 52,97\%). Cette teneur en glucide élevée pourrait s'expliquer par l'ajout des épices et ingrédients issus de la production du Kilishi riche en glucide tels que le tourteau d'arachide, le sucre, les diverses épices moulues ajoutés au fond de sauce nature pour la production des fonds de sauces concentrés et séchées.

Quant à la qualité microbiologique des fonds de sauces, les charges en flore mésophile aérobie totale, en coliformes totaux et en $S$. aureus en UFC/g de nos échantillons de fonds de sauces natures et concentrés sont inférieures à la limite recommandée par le Ministère de la Santé du Luxembourg (2018) pour les produits de charcuterie cuits qui sont de $3 \times 10^{5} \mathrm{UFC} / \mathrm{g}$ pour la flore mésophile aérobie totale, de $10^{3}$ $\mathrm{UFC} / \mathrm{g}$ pour les coliformes et de $10^{2} \mathrm{UFC} / \mathrm{g}$ pour $S$. aureus. Par contre celles des fonds de sauces séchés sont supérieures à la limite recommandée par le Ministère de la Santé du Luxembourg (2018) pour ces produits, les fonds de sauces séchés sont donc impropres à la consommation humaine.

Egalement les résultats des analyses microbiologiques effectuées sur les fonds de sauces concentrés de types Kilishi après quatre mois de conservation ont montré que la charge microbiologique de ces fonds de sauces sont identiques à celle analysée au premier jour de production. On peut donc dire que ces deux formulations de fonds de sauces concentrés se conservent bien à la température ambiante. La bonne qualité microbiologique des fonds concentrés et leur stabilité microbiologique après quatre mois de conservation pourrait être due à l'efficacité de la stérilisation et à l'application des règles de bonnes pratiques d'hygiène et de fabrication pendant la production des fonds de sauces concentrés de type Kilishi. La stérilisation des fonds de sauces concentrés a eu un effet dommageable sur la flore microbienne et les spores contrairement aux types de traitements thermiques appliquées (séchage, rôtissage, fumage) au fonds de sauce séchés de type Kilishi qui n'aurait pas permis de réduire considérablement la charge microbienne a un niveau acceptable. S'agissant des saucisses produites dans le but d'évaluer la qualité sensorielle des fonds de sauces, les résultats des analyses microbiologiques effectuées sur les échantillons de saucisses ont montré que la charge en UFC/g de la flore mésophile aérobie totale a varié de moins de 10 à $7,1.10^{2}$ dans les trois formulations de saucisses. Quant aux charges en UFC/g des coliformes totaux, des levures et moisissures, des $B$. cereus et $S$. aureus, elles étaient de moins de 10 chacune. Les charges en flore mésophile aérobie totale, en coliformes totaux et en $S$. aureus en UFC/g de nos échantillons de saucisses sont inférieures à la limite recommandée par le Ministère de la Santé du Luxembourg (2018) pour les produits de charcuterie cuits qui sont de $3,0.10^{5} \mathrm{UFC} / \mathrm{g}$ pour la flore mésophile aérobie totale, de $10^{3} \mathrm{UFC} / \mathrm{g}$ pour les coliformes et de $10^{2} \mathrm{UFC} / g$ pour $S$. aureus. La bonne qualité microbiologique des saucisses s'expliquerait par l'efficacité du traitement appliquée au saucisses (rôtissage, fumage des saucisses) dans le séchoir à gaz qui aurait permis de réduire considérablement la charge microbienne et à l'application des règles de bonnes pratiques d'hygiènes et de fabrications lors de la production des saucisses étudiées. Le même constat a été fait par Tiendrébéogo et al. (2016) que l'utilisation du séchoir à gaz pour les traitements thermiques et l'application des BPH\&BPF réduiraient considérablement les microorganismes pathogènes dans le Kilishi.

Egalement les résultats des analyses physicochimiques ont montré que les saucisses étaient toutes de bonne qualité nutritionnelle. En effet, nos valeurs en matières grasses (3,38 à $9,79 \%$ ) sont toutes inferieures à la limite fixée par le code d'usage de la charcuterie, de la salaison et des conserves de viandes (2016), qui est de $30 \%$. L'excès de matières grasses doit, cependant, être évité dans nos choix de consommation alimentaire, car leur 
consommation élevée peut entraîner certaines maladies cardiovasculaires et accroître le risque de prise de poids à cause de la richesse en calorie (Smith-Schneider et al., 1992). Egalement, les valeurs en HPD des saucisses $(58,10$ à $67,03 \%)$ étudiées sont toutes inférieures à la limite fixée par le code d'usage de la charcuterie, de la salaison et des conserves de viande (2016) qui est de $80 \%$. Ces valeurs en HPD permettent une bonne conservation de nos saucisses. En effet, l'HPD dépend des quantités d'eau ou de matières riches en eau ajoutées. Leurs limites sont fixées pour les produits de charcuterie et de salaison en fonction de la technologie; plus le produit est cuit ou séché, plus l'HPD est basse. Si, au contraire, il y a plus d'addition d'eau, glace ou de substances riches en eau, alors l'HPD est plus élevée (CTSCV, 2016). En comparant nos résultats en protéines $(27,50$ à $32,67 \%)$ avec les autres types de saucisses cuites, nous constatons que nos formulations sont très riches en protéines et les valeurs en protéines de nos formulations sont supérieures à celle des saucisses de Francfort et de Strasbourg qui sont, respectivement, de 12,7 et de $13,3 \mathrm{~g}$ pour $100 \mathrm{~g}$ de produit (Frentz et Juillard, 2003 ; CTSCCV, 2016).

Les résultats de l'analyse sensorielle ont montré que les scores des échantillons de saucisses ont varié de 4,04 à 4,54 soit une note d'appréciation moyenne de 4,32 ce qui correspond au goût agréable. Le test de Friedman $(\mathrm{P}<0,05)$ a montré une différence significative entre les saucisses issues des fonds concentrés de type Kilishi issus de la formulation 2 et le témoin. Parmi toutes ces formulations, les deux formulations de saucisses fabriquées avec ajout des fonds de sauces concentrés de type Kilishi ont été les plus appréciées et jugées agréable par le panel de dégustateurs. Par contre la formulation de saucisses qui a été la moins apprécié par le panel était le témoin qui contenait pas de fonds de sauces. Il ressort des résultats que l'ajout des fonds de sauces dans la production des saucisses améliorerait le goût des saucisses. Les fonds de sauces concentrés produits dans cette étude seraient donc des exhausteurs de goût d'origine naturelle.

\section{Conclusion}

Les travaux réalisés dans le cadre de la présente étude ont permis de valoriser les os issus de la production du Kilishi en des fonds de sauce (concentrés et séchés). Il ressort des résultats des analyses physicochimiques, que les fonds séchés constituent une bonne source d'énergie et de matières minérales par rapport aux fonds concentrés. L'analyse microbiologique a montré que les fonds concentrés étaient de bonnes qualités comparées aux fonds séchés qui présentaient des valeurs élevées au niveau de certains paramètres comme les $B$. cereus et la flore totale. L'utilisation des fonds de sauce concentrés pour la production des saucisses a donné des résultats satisfaisants en analyses physicochimiques et microbiologiques. Les saucisses issues des fonds concentrés ont été plus appréciées suite au test hédonique que les saucisses témoin qui ont été produites sans fonds de sauce. Les résultats révèlent que les fonds de sauces concentrés étudiés seraient des exhausteurs de goût d'origine naturelle qui pourraient être utilisés à la place des exhausteurs de goût de type monosodique qui présenteraient des dangers pour la santé des consommateurs en cas d'excès de consommation. L'étude de stabilité des fonds de sauce concentrés a également montré qu'ils se conservent bien à température ambiante. La présente étude est une contribution à la diversification des exhausteurs de goût d'origine naturelle.

\section{CONFLITS D'INTERETS}

Les auteurs déclarent n'avoir aucun conflit d'intérêts quant à la réalisation de cette étude et la rédaction de cet article.

\section{CONTRIBUTIONS DES AUTEURS}

Tous les auteurs ont participé à la réalisation de cette étude et à la rédaction de cet article.

\section{REMERCIEMENTS}

Les remerciements vont à l'endroit du personnel du Département Technologie Alimentaire (DTA) de l'Institut de Recherche 
en Sciences Appliquées et Technologies (IRSAT) pour leur collaboration.

\section{REFERENCES}

AOAC. 1990. Official Methods of Analysis (15th Edn). Association of Official Analytical Chemists: Washington DC.

Atwater WO, Benedict FG. 1899. Experiments on the metabolism of matter and energy in the human body. Agr. off. Expt. Stas. Bul 69 -rev. eg: USA.

Badau MH, Igene J0, Collisson EK, Nkama I. 1997. Studies on product, physicochemical and sensory properties of a standard Kilishi ingrédient mix powder. International Journal of Food Sciences and Nutrition, 48 : 165-168.

Bello MM, Tekwata YD. 2015. Nutritive properties and consumer acceptance of solar tent and sun dried Kilishi from Bony-Tongue (Heterotis, niloticus, Cuvier 1829). In a Semi-Arid Zone of Nigeria. Nigerien Journal of Fisheries, 12(1): $\quad 801-808 . \quad$ Doi : https://www.researchgate.net/publication/ 303912451

Boutry C. 2010. Influence d'une supplémentation en monosodium glutamate sur la physiologie gastrointestinale et le métabolisme chez le rat et l'homme. Thèse de doctorat, 'Institut des Sciences et Industries du Vivant et de l'Environnement, Paris, p.146.

Chukwu O, Imodiboh IL. 2009. Influence of Storage Conditions on Shelf-life of Dried Beef Product (Kilishi). World Journal of Agricultural Sciences, 5(1) : 34-39.

Code d'usage de la Charcuterie, de la Salaison et des Conserves de Viande. 2016. Réglementation et usage. Maison Alfort : Paris.

Deppenweiler A. 2014. Le glutamate monosodique comme exhausteur de goût confiance ou méfiance. Thèse d'état en pharmacie, Université Victor Segalen Bordeaux 2, Bordeaux, p.119.

Derra A. 2021. Mise au point technologique et caractérisations microbiologique, sensorielle des saucisses produites à partir de la viande de bœuf en utilisant les épices et ingrédients locaux. Mémoire de Master, Université Joseph Ki Zerbo, Ouagadougou, p. 67.

Frentz JC, Julliard M. 2003. Encyclopédie de la Charcuterie. MAE-ERTI (Ed) : Paris.

Igene OJ, Farouk MM. 1990. Preliminary Studies on the tradition Processing of Kilishi. J. Sci Food Agric., 50 : 89-98.

Iheagwara MC, Okonkwo TM. 2016. Effect of Processing Techniques on the Microbiological Quality of Kilishi - A traditional Nigerian Dried Beef product. Journal of Meat Science and Technology, 4(1): 11-17. DOI : https://www.researchgate.net/publication/ 332531165.

Isah OA, Okubajo AO. 2012. Effect of various Additives on proximate composition and acceptability of Kilishi made from semitendinose Muscle of White Fulani Cattle. The Pacific Journal of Science and Technology, 13(1) : 506-511.

Jega IJ, Magawata I, lpinjolu JK, Jibir M. 2013. Evaluation of slurry formulations for Kilishi processing of Africain lungfish (Protopterus annectens, Owen). Pakistan Journal of Nutrition, 12(7) : 673-677.

Kalilou S, Collignan A, Zakhia N. 1998. Optimizing the traditional processing of Beef into Kilishi. Meat Science, 50(1) : 21-32.

Kurihara K. 2009. Glutamate: from discovery as a food flavor to role as basic taste. The American Journal of Clinical Nutrition, 90(3) : 719-722.

Linder M. 1996. Optimisation d'un procède de valorisation de coproduits d'abattage par Hydrolyse enzymatique: propriétés fonctionnelles et nutritionnelles des hydrolysats. Thèse de doctorat, Université de Loraine, Loraine, p. 245.

Mgbemere VN, Akpapunam MA, Igene JO. 2011. Effect of groundnut flour substitution on Yield, Quality and Storage Stability of Kilishi-A Nigerian Indigenous Dried Meat product. African Journal of Food Agriculture, Nutrition and Developpement, 11(2) : 4718-4738.

Ministère de la Santé du Grand-Duché de Luxembourg. 2018. Critères 
microbiologiques applicables aux denrées alimentaires lignes directives pour l'interprétation des résultats. Edition Août 2018 : Grand-Duché de Luxembourg.

Musonge P, NjolaiI EN. 1994. Drying and Infusion During the traditionnel Processing of Kilishi. Journal of Food Engineering, 23 : 159-168.

Norme Française NF ISO 7932. 1993. Directives générales pour le dénombrement de Bacillus cereus, technique par comptage des colonies à 30 ${ }^{\circ} \mathrm{C}$. AFNOR : Paris.

Norme Française NF ISO 7954. 1988. Directives générales pour le dénombrement des levures et moisissures, technique par comptage des colonies à 25 ${ }^{\circ} \mathrm{C}$. AFNOR : Paris.

Norme française V03- 050. 1970. Directives générales pour le dosage de l'azote avec minéralisation selon la méthode de Kjeldahl. AFNOR : Paris.

Norme Internationale ISO 4832. 2006. Microbiologie des aliments- Méthode horizontale pour le dénombrement des coliformes - Méthode par comptage des colonies obtenues à $37{ }^{\circ} \mathrm{C}$. AFNOR : Paris.

Norme Internationale ISO 659.1998. Graines oléagineuses. Détermination de la teneur en huile. AFNOR : Paris.

Norme internationale ISO 665. 2000. Graines oléagineuses. Détermination de la teneur en eau et en matières volatiles. AFNOR : Paris.

Norme Internationale ISO 6887-1. 1999. Microbiologie des aliments. Préparation des échantillons, de la suspension mère et des dilutions décimales en vue de l'examen microbiologique-Partie 1 : Règles générales pour la préparation de la suspension mère et des dilutions décimales. AFNOR : Paris.
Norme Internationale ISO 4832-2. 2006. Microbiologie des aliments-Méthode horizontale pour le dénombrement des Staphylococcus aureus à caogulase positive et autres espèces). Partie 2 : technique utilisant le milieu gélosé au plasma de lapin et au fibrinogène. AFNOR, Paris.

Nout MJR, Notermans S, Rombouts FM. 1998. Effect of environmental conditions during soya-bean fermentation on growth of Staphylococcus aureus and production and thermal stability of anterotoxins A and B. International Journal of Food Microbiology, 7(4) : 299-309. DOI : 10.1016/0168-1605(88)90056-6

Ogunsola OO, Omojola AB. 2008. Qualitative evaluation of Klishi prepared from beef and pork. African journal of Biotechnology, 7(11) : 1753-1758.

Olusola OO, Okubanjo AO, Omojola AB. 2012. Nutritive and Oganoleptic Characteristics of Kilishi as Affected by Meat type and Ingredient Formulation. $J$ Animal. Prod., 2(5) : 221-232.

Poulain JM, Frentz JC, Tourteaux C. 2017. Livre du Compagnon Charcutier Traiteur. Delagrave Edition: Paris.

Smith-Schneider LM, Sigman-Grant MJ, KrisEtherton PM. 1992. Dietary Fat reduction strategies. J. Am Diet Assoc., 92: 34-38.

Tiendrébeogo SCW, Sawadogo-Lingani H, Kabore D, Zida-Ouedraogo K, Pare A, Samandoulougou S, Dicko MH. 2016. Evaluation de l'impact du séchoir à gaz BB équipement sur la qualité du Kilichi. Revue Science et Technique, Spécial horssérie $2: 191-203$.

Watts BM, Ylimaki GL, Jeffery LE, Elias LG. 1991. Méthodes de Base pour l'Evaluation Sensorielle des Aliments. Ont. CRDI : Ottawa. 